\title{
Valores y Personalidad como Predictores del Aprecio por las Prácticas Sostenibles en Emprendedores Sociales y Comerciales de Lima-Perú
}

\section{Values and Personality as Predictors of the Appreciation for Sustainable Practices in Social and Commercial Entrepreneurs of Lima-Peru}

\author{
Angela Vera Ruiz ${ }^{1}$, Agustín Espinosa ${ }^{1}$ y María Ángela Prialé Valle ${ }^{2}$ \\ ${ }^{1}$ Departamento de Psicología, Pontificia Universidad Católica del Perú \\ 2 Departamento de Administración, Universidad del Pacífico
}

\begin{abstract}
Este estudio buscó analizar la capacidad predictiva de los valores (Schwartz, 1992) y la personalidad (Goldberg, 1990) en el aprecio por prácticas sostenibles (APS). El APS es una dimensión actitudinal hacia la sostenibilidad, medida con una escala desarrollada específicamente para esta investigación y aplicada en el contexto de la actividad emprendedora. Asimismo, se analizó la capacidad clasificatoria del APS para la identificación de perfiles de emprendedores, según el tipo de emprendimiento que realizan (social versus comercial), y se comparó con la capacidad clasificatoria de los valores y la personalidad. Para tales fines, se utilizó una muestra no probabilística intencionada de emprendedores de Lima Metropolitana, previamente identificados como sociales $(n=135)$ o comerciales $(n=198)$. Una regresión lineal múltiple muestra que el APS es predicho por valores de autotrascendencia y por los rasgos de agradabilidad y apertura intelectual. Un análisis de regresión logística binaria muestra que la capacidad clasificatoria del APS alcanza un 68,8\% de efectividad en la identificación correcta de casos, según tipo de emprendimiento, versus un $76 \%$ de los valores y la personalidad en conjunto. Sin embargo, la capacidad clasificatoria del APS es superior a la de los valores y la personalidad cuando se analizan las dimensiones de estos constructos por separado. Se discuten la capacidad clasificatoria del APS para la detección de emprendedores de orientación social como un criterio parsimonioso, alternativo y complementario a los perfiles de valores y personalidad y las implicancias del mismo en la comprensión de emprendimientos innovadores y sostenibles.
\end{abstract}

Palabras clave: aprecio por prácticas sostenibles, emprendimiento social, personalidad, valores

This study sought to analyze the predictive capacity of values (Schwartz, 1992) and personality (Goldberg, 1990) regarding the appreciation of sustainable practices (APS). The APS is a dimension that indicates a person's attitude toward sustainability, measured with a scale developed specifically for this study and applied in the context of entrepreneurial activity. Likewise, the classification capacity of the APS regarding the identification of entrepreneur profiles by type of entrepreneurship (social versus commercial) was analyzed and then compared with the classification capacity of values and personality. To this end, an intentional non-probability sample of entrepreneurs in Metropolitan Lima, previously classified as social $(n=135)$ or commercial $(n=198)$, was used. A multiple linear regression shows that the APS is predicted by self-transcendence values and the personality traits of agreeableness and intellectual openness. A binary logistic regression analysis shows that the classificatory capacity of the APS reaches $68.8 \%$ in the correct identification of cases, according to the type of venture undertaken, versus $76 \%$ for values and personality as a whole. However, the classificatory capacity of the APS is superior to that of values and personality when the dimensions of these constructs are analyzed separately. The discussion covers the classificatory capacity of the APS for the detection of socially oriented entrepreneurs as a parsimonious criterion, alternative and complementary to values and personality profiles, along with the implications of this construct for the understanding of innovative and sustainable entrepreneurship models.

Keywords: appreciation of sustainable practices, social entrepreneurship, personality, values

\section{Angela Vera Ruiz (D) https://orcid.org/0000-0002-8491-3979 \\ Agustín Espinosa iD https://orcid.org/0000-0002-2275-5792}

María Ángela Prialé Valle (D) https://orcid.org/0000-0003-4914-6388

La correspondencia relativa a este artículo debe ser dirigida a Angela Vera, Departamento de Psicología, Pontificia Universidad Católica del Perú, Avenida Universitaria 1801, San Miguel, Lima, Perú. Email: averar@pucp.pe 
En el análisis de la actividad emprendedora, el aprecio por prácticas sostenibles (APS) es propuesto como una dimensión actitudinal que explica la necesidad de desarrollar y emplear prácticas y modelos de producción que mitiguen los problemas sociales derivados de sistemas centrados estrictamente en el lucro, siendo el motor de emprendimientos de naturaleza social (İrengün \& Arıkboğa, 2015; Kuckertz \& Wagner, 2010; Mair \& Martí, 2006; Vera Ruiz et al., 2019, 2020). De acuerdo a Nga y Shamuganathan (2010), el APS es una dimensión importante en el comportamiento de emprendedores con orientación social y tiene su fundamento en la convicción de que las acciones emprendedoras equilibradas y respetuosas con el entorno, pueden mejorar y modificar el sistema circundante y resolver problemas sociales y/o ambientales urgentes y desatendidos (Abu-Saifan, 2012; Kneiding \& Tracey, 2009; Leisinger, 2007; Zhang \& Swanson, 2014).

Por tal motivo, el APS implica asumir una conciencia moral y reflexiva sobre las propias acciones emprendedoras (Leisinger, 2007; Mair \& Noboa, 2006; Whitman, 2011), reconociendo que individuos y organizaciones son parte de un ecosistema en el que pueden y deben generarse nuevas formas de relación con el entorno, caracterizadas por ser social y ambientalmente sustentables, responsables, equilibradas, inclusivas y justas (Cartwright \& Craig, 2006; Savitz \& Weber, 2006). Asimismo, estas actividades deben estar orientadas por la empatía y el interés en el prójimo, en aras de alcanzar el bienestar social (Mair \& Noboa, 2006; Martin \& Osberg, 2007).

Desde la psicología social, es razonable afirmar que el emprendimiento en general y el APS en particular podrían estar relacionados con los valores y la personalidad (Vera Ruiz et al., 2019). De manera específica, diversos estudios relacionados a los valores, por una parte, y a la personalidad, por otra, ponen en consideración diferencias psicológicas significativas entre los perfiles de emprendedores sociales versus los emprendedores comerciales - aquellos cuyos emprendimientos se orientan, en primera instancia, a la creación de valor económico (Young, 2006) - y permiten, de manera conceptual, asociarlos con sus particulares visiones de negocios y del APS (Austin et al., 2006; Nga \& Shamuganathan, 2010; SastreCastillo et al., 2015).

En esta línea, a través del estudio de las características psicológicas de los emprendedores sociales, se ha identificado en ellos atributos similares a los de emprendedores comerciales, como son apertura mental, capacidad de innovación, planeación, toma de riesgos, ejecución y logro de resultados, liderazgo, alta autoeficacia y capacidad para generar retornos financieros, entre otros (Austin et al., 2006; Guzmán Vásquez \& Trujillo Dávila, 2008; İrengün \& Arıkboğa, 2015; Nga \& Shamuganathan, 2010; Young, 2006). Sin embargo, también se ha corroborado que los emprendedores sociales tienen mayor visión social, una mayor propensión a la preocupación por los otros y un alto sentido de responsabilidad sobre una realidad que buscan transformar con sus acciones (Caballero et al., 2014; Chell, 2008; Levie \& Hart, 2011; Mair \& Noboa, 2006; Sastre-Castillo et al., 2015; Seelos \& Mair 2005; Sullivan Mort et al., 2003). Los estudios también señalan que, en la búsqueda del cambio social a través de la acción emprendedora (Mair \& Noboa, 2006), estarían involucrados procesos cognitivos de valoración de prácticas productivas sostenibles en la creación de modelos innovadores de negocio (Bruyat \& Julien, 2001).

Con relación a los valores, para el presente trabajo se consideró el modelo de valores individuales de Schwartz (1992), debido a su validez transcultural, corroborada en diferentes estudios en el contexto peruano y latinoamericano (e.g., Beramendi et al., 2013; García Anchorena et al., 2016; Imhoff \& Brussino, 2013), y a su pertinencia explicativa avalada por estudios sobre emprendimiento (García Villaverde \& Valencia de Lara, 2009; Moriano et al., 2001; Ormeño Coronado, 2014; Sastre-Castillo et al., 2015). Desde esta propuesta, los valores se definen como metas deseables de existencia que varían de acuerdo con la importancia que subjetivamente se les asigna y que orientan la evaluación y selección de comportamientos, personas o eventos deseables, trascendiendo situaciones específicas (Schwartz, 1992; Schwartz \& Bilsky, 1987).

El modelo de Schwartz propone la existencia de 10 dimensiones axiológicas universales que se pueden agrupar en cuatro metas motivacionales, a su vez comprendidas en dos dimensiones bipolares ortogonales. En la primera dimensión, el conjunto de valores de autotrascendencia (benevolencia y universalismo) se contrasta con los valores de autopromoción (poder, logro y hedonismo). El primero alude a los intereses del individuo en función del bienestar de los demás y el segundo se refiere a los intereses en función de sí mismo. En la segunda dimensión, los valores de conservación (tradición, conformidad y seguridad) se contrastan con los valores de apertura al cambio (autodirección, estimulación y hedonismo) (Schwartz, 1992).

El análisis axiológico en los estudios sobre el emprendimiento busca caracterizar los valores que 
sustentan el comportamiento emprendedor (Cater III et al., 2017; Kecharananta \& Barker, 1999; Marulanda Valencia et al., 2014; Moriano et al., 2001; Ormeño Coronado, 2014; Sastre-Castillo et al., 2015). Los resultados de estas investigaciones permiten establecer que los emprendedores tienen un perfil axiológico diferente al de otras ocupaciones (Kecharananta \& Baker, 1999). En el contexto particular de Latinoamérica, trabajos con el modelo de Schwartz han encontrado que, en general, los emprendedores comerciales están orientados hacia valores individualistas de autopromoción y apertura al cambio, lo que implica perseguir el éxito personal y demostrar competencia según estándares sociales de poder y logro de manera activa, independiente e innovadora (García Villaverde \& Valencia de Lara, 2009; Marulanda Valencia et al., 2014; Moriano et al., 2001; Ormeño Coronado, 2014). El hedonismo conlleva satisfacer el deseo de hacer algo que realmente se disfruta, a través de la creación de la propia empresa (Moriano et al., 2001), mientras que valores colectivistas, como los de tradición, estarían inversamente relacionados con las intenciones y el éxito emprendedor (García Villaverde \& Valencia de Lara, 2009; Moriano et al., 2001).

Por otra parte, estudios con emprendedores sociales evidencian que estos presentan valores de conservación, como la seguridad y la tradición (Ormeño Coronado, 2014), y valores de autotrascendencia, como la benevolencia y el universalismo (Abhi, 2017; Ormeño Coronado, 2014; Sastre-Castillo et al., 2015), asumiendo un posicionamiento ético altamente altruista, orientado por la búsqueda del bienestar social (Cater III et al., 2017; Ruskin et al., 2016).

Adicionalmente, cuando los valores se analizan en conjunto con la personalidad, destaca su mutua influencia, ya que las personas buscan consistencia entre sus valores y sus comportamientos, por lo que autorregulan la manifestación de ciertos rasgos en función de la correspondencia con sus creencias centrales y, a su vez, incrementan la valoración de sus objetivos cuando exhiben consistentemente un rasgo en su comportamiento (Carver \& Scheier, 1981; Roccas et al., 2002; Rokeach, 1973; Schwartz, 1994).

La personalidad — definida como un patrón único y particular de pensamientos, sentimientos y conductas del individuo, persistentes a través del tiempo y las situaciones (McCrae \& Costa Jr., 1992; Morris \& Maisto, 2011) - también ha sido empleada en la caracterización de los emprendedores. En esa tarea, el modelo conceptual de los cinco grandes (big five) de la personalidad ha sido ampliamente utilizado (Antoncic et al., 2015; Zhao \& Seibert, 2006).

Este modelo considera cinco grandes dimensiones o rasgos: (a) Extraversión: comprende características de asertividad, dominancia, energía, actividad y una tendencia a la pertenencia a grandes grupos (McCrae \& Costa Jr., 1990; Zhao \& Seibert, 2006); (b) Agradabilidad: rasgo asociado a la calidad de los vínculos establecidos con otros a partir de la confianza, altruismo, cuidado y cooperación; (c) Consciencia: comprende características como alto sentido de organización, persistencia y trabajo duro para alcanzar objetivos (Zhao \& Seibert, 2006); (d) Neuroticismo: representa características vinculadas a la ansiedad, hostilidad, depresión e impulsividad, en contraposición a la adaptabilidad y la estabilidad emocional (McCrae \& Costa Jr., 1990; Zhao \& Seibert, 2006) y (e) Apertura a la experiencia/al intelecto: comprende características vinculadas a la curiosidad intelectual y a la búsqueda de nuevas experiencias o ideas, creatividad y pensamiento divergente (Antoncic et al., 2015; McCrae \& Costa Jr., 1990; Zhao \& Seibert, 2006).

Diferentes estudios han identificado que altos puntajes en extraversión, consciencia, estabilidad emocional y apertura son comunes en los emprendedores en general (Brandstätter, 2011; Luca, 2017; Nga \& Shamuganathan, 2010; Zhao \& Seibert, 2006). Por su parte, un bajo puntaje en agradabilidad resulta ser el predictor más consistente de la carrera emprendedora sobre los otros cuatro grandes rasgos (Brandstätter, 2011; Caballero et al., 2014; Zhao \& Seibert, 2006), debido a que velar por el propio interés para conducir negocios sólidos, en un mercado competitivo, resulta incompatible con la consideración por otros, si es que esto amenaza los intereses y la toma de decisiones empresariales (Caballero et al., 2014; Liang et al., 2015; Wood, 2012; Zhao \& Seibert, 2006).

No obstante, distintos estudios demuestran que los emprendedores sociales presentan niveles de agradabilidad significativamente altos, debido a su orientación por el bien común y el cuidado de las necesidades, urgentes y no atendidas, de otros (Caballero et al., 2014; Liang et al., 2015; Mair \& Noboa, 2006; Nga \& Shamuganathan, 2010; Wood, 2012). Este hallazgo pone de vuelta la mirada en los resultados de investigaciones sobre perfiles axiológicos y reafirma la necesidad de considerar la articulación entre las dos variables, para evaluar consistencias en los perfiles de emprendedores que tienen orientaciones sociales.

Si a los temas señalados se suman las especificidades contextuales del Perú — determinantes para el desarrollo de los distintos tipos de emprendimiento (Bikse et al., 2015; Gurrieri, 2013; Leadbeater, 1997; 
Sastre-Castillo et al., 2015)—, entre las que destacan: la informalidad de la economía apostada en prácticas emprendedoras por necesidad (Global Entrepreneurship Research Association, 2017; Serida et al., 2010), el conservadurismo, la dominancia, el colectivismo (Cueto et al., 2017) y la alta orientación a valores materialistas (Castaman Díaz, 2017) que reduce la probabilidad de perseguir fines autotrascendentes (Inglehart, 1981), resulta de interés comprender las condiciones y características psicosociales que favorecen la presencia de niveles más altos de APS en emprendedores.

Adicionalmente, a nivel práctico, estudiar las relaciones entre APS, valores y personalidad con el emprendimiento resulta crucial para identificar y desarrollar perfiles de emprendedores capaces de viabilizar emprendimientos innovadores que equilibren el valor social y comercial y se orienten al bienestar social, a la preservación ambiental y a la productividad sostenible (Bikse et al., 2015; Brandsen \& Karré, 2011; Cope, 2005; García Villaverde \& Valencia de Lara, 2009; Shaver \& Scott, 1992; Smith et al., 2017; Zahra et al., 2009), ya que el conocimiento de estos constructos puede ser insumo para el desarrollo de programas de formación ad hoc que nazcan desde el sector privado e, incluso, público.

Por ello, el presente estudio tuvo dos objetivos. El primero fue analizar la capacidad predictiva de los valores y la personalidad sobre el APS para identificar y confirmar la especificidad de perfiles psicosociales con mayor disposición hacia la sostenibilidad emprendedora. El segundo fue determinar la capacidad clasificadora del APS en la identificación de perfiles de emprendedores según el tipo de emprendimiento que realizan (social versus comercial) y compararla con la capacidad clasificadora de los valores y la personalidad en la misma clasificación de perfiles.

En relación con el primer objetivo enunciado, y en función de la literatura revisada, se formularon las siguientes hipótesis:

H1. Los valores de autotrascendencia predicen directamente el APS.

H2. Los valores de conservación predicen directamente el APS.

H3. Los valores de apertura al cambio predicen directamente el APS.

H4. Los valores de autopromoción predicen inversamente el APS.

H5. El rasgo de personalidad de agradabilidad predice directamente el APS.

H6. El rasgo de personalidad de apertura predice directamente el APS.

H7. Los rasgos de personalidad de consciencia, extraversión y neuroticismo no predicen el APS.

En cuanto al segundo objetivo enunciado, se hipotetizó lo siguiente:

H8. El APS tiene una buena capacidad en la clasificación de emprendedores sociales y comerciales, siendo su prevalencia mayor en los primeros.

H9. El APS tiene una mejor capacidad que los valores y la personalidad en la clasificación de emprendedores, según el tipo de emprendimiento que realicen (social versus comercial).

\section{Método}

El estudio desarrollado fue comparativo-correlacional.

\section{Participantes}

El estudio contó con 333 participantes de Lima, Perú, seleccionados a través de un muestreo no probabilístico intencional y distribuidos en dos grupos. El primer grupo fueron 135 emprendedores sociales (40,5\% del total de la muestra), contactados usando la información de una base de datos compuesta por 384 emprendedores sociales. Dicha base de datos fue construida rigurosamente por investigadores de una universidad de Lima, quienes emplearon información brindada por entidades que promueven el emprendimiento social. La tasa de respuesta final de ese grupo de emprendedores fue de un 35\%. El segundo grupo fueron 198 empresarios comerciales (59,5\% del total de la muestra), quienes fueron contactados tras la elaboración de una base de datos basada en el registro de empresas en cámaras de comercio, patronatos y directorios empresariales de Lima. Esta base contaba con 880 contactos, siendo la tasa de respuesta final un $22,5 \%$.

Las edades de los participantes estaban comprendidas entre los 18 y 80 años $(M=38,56, D E=12,59)$ y $49,8 \%$ de las participantes eran mujeres. Los niveles socioeconómicos autorreportados oscilaron entre bajo/medio-bajo $(14,36 \%)$, medio $(65,54 \%)$, medio-alto/alto $(20,10 \%)$. No se reportaron diferencias del promedio de edad del grupo de emprendedores sociales $(M=38,16, D E=12,47)$ versus emprendedores 
comerciales $(M=39,25, D E=12,68), t(331)=-0,78, p=0,438$. Por otro lado, no se hallaron diferencias en el sexo, pero sí en el nivel socioeconómico reportado, observándose en los emprendedores sociales una prevalencia de perfiles socioeconómicos concentrados en los extremos de clase alta o clase baja - con prevalencia en la primera-, en comparación con los emprendedores comerciales, en los que el perfil socioeconómico tiende a estar más concentrado en los sectores de clase media (ver Tabla 1).

Tabla 1

Descriptivos y Comparaciones de las Características Sociodemográficas de los Participantes por Tipo de Emprendimiento (Sociales Versus Comerciales)

\begin{tabular}{|c|c|c|c|c|c|}
\hline \multirow{2}{*}{ Variable } & \multirow{2}{*}{$\begin{array}{l}\text { Emprendedores } \\
\text { sociales }\end{array}$} & \multirow{2}{*}{$\begin{array}{l}\text { Emprendedores } \\
\text { comerciales }\end{array}$} & \multicolumn{3}{|c|}{ Comparación intergrupo } \\
\hline & & & $\chi^{2}$ & $g l$ & $p$ \\
\hline Sexo & $\begin{array}{l}\text { Hombres: } 49,6 \% \\
\text { Mujeres: 50,4\% }\end{array}$ & $\begin{array}{l}\text { Hombres: } 50,5 \% \\
\text { Mujeres: } 49,5 \%\end{array}$ & 0,025 & 1 & 0,875 \\
\hline $\begin{array}{l}\text { Nivel } \\
\text { socioeconómico }\end{array}$ & $\begin{array}{l}\text { Bajo/Medio-bajo }=21,6 \% \\
\text { Medio }=44,0 \% \\
\text { Medio-alto/Alto }=34,4 \%\end{array}$ & $\begin{array}{l}\text { Bajo/Medio-bajo }=16,7 \% \\
\text { Medio }=66,7 \% \\
\text { Medio-alto/Alto }=16,7 \%\end{array}$ & 18,654 & 2 & 0,001 \\
\hline
\end{tabular}

Nota. $n=333$.

\section{Instrumentos}

\section{Escala de Personalidad de Goldberg (1990)}

La escala mide cinco grandes factores de personalidad (Goldberg, 1990) en un diferencial semántico compuesto por 40 pares de adjetivos opuestos, ocho para cada uno de los cinco factores: Extraversión (e.g., pasivo-activo), Agradabilidad (e.g., frío-cálido), Consciencia (e.g., desorganizado-organizado), Neuroticismo (e.g., seguro-inseguro) y Apertura Intelectual (e.g., inculto-culto). Los pares van del 1 al 7, donde los valores con mayor proximidad a 1 se relacionan con el primer adjetivo, y los más cercanos a 7 , con el segundo. Para el presente estudio, se usó la versión adaptada por Calderón Prada (2003) en una muestra de adultos de zonas urbano-marginales de Lima, en la que se corroboró la validez de constructo de la escala. Esta versión fue utilizada posteriormente por Espinosa et al. (2011) en muestras urbanas de niveles socioeconómicos medios y altos de Lima con niveles de confiabilidad aceptables. En el presente estudio, la escala cuenta también con niveles aceptables de consistencia interna, evaluada con alfa de Cronbach, para las dimensiones Extraversión $(\alpha=0,68)$, Agradabilidad $(\alpha=0,52)$, Consciencia $(\alpha=0,54)$ y Apertura Intelectual $(\alpha=0,69)$ y baja consistencia interna para la dimensión Neuroticismo $(\alpha=0,47)$. Con relación a los coeficientes de consistencia interna reportados, se asumió que estos resultaban aceptables para el trabajo estadístico. Dadas las características de la escala, se espera cierta estabilidad, poca variabilidad y un sesgo de autopresentación positiva en las respuestas, lo que podría reducir la confiabilidad en las medidas, por lo que, de acuerdo con el criterio observado en el meta-análisis de Mezulis et al. (2004), coeficientes de consistencia interna cuyos valores van de 0,50 a 0,70 se podrían considerar moderados y, de acuerdo con los autores citados, su desempeño estadístico no se diferencia significativamente de medidas con mayores niveles de confiabilidad. Este criterio de interpretación fue aplicado para todas las escalas con un componente de autopresentación.

\section{Cuestionario de Valores Personales de Schwartz (1992)}

Se trata de una escala que consta de 40 enunciados que miden perfiles axiológicos o valores personales. Ante esta escala, un participante debe contestar en qué medida se parece a la persona descrita en cada ítem. La opción de respuesta es una escala tipo Likert entre 1 (no se parece a mî) y 6 (se parece mucho a mî). El cuestionario permite extraer puntuaciones para 10 tipos de valores (poder, logro, hedonismo, estimulación, autodirección, universalismo, benevolencia, tradición, conformidad, seguridad) que están 
organizados en cuatro dimensiones, cada una de ellas representada por 10 ítems: Autotrascendencia (e.g., "Es muy importante para él/ella ayudar a la gente que le/la rodea"), Autopromoción (e.g., "Para él/ella es importante ser respetado por la gente"), Conservación (e.g., "Le importa vivir en lugares seguros") y Apertura al Cambio (e.g., "Tener ideas nuevas y ser creativo/a es importante para él/ella") (Schwartz, 1992; Schwartz \& Bilsky, 1987). Para la presente investigación se utilizó la versión validada por Herrera y Lens (2003) en una muestra peruana, observándose un buen funcionamiento psicométrico de la misma en otros estudios realizados en este país (Espinosa et al., 2011). En el presente estudio se utilizaron para los análisis correspondientes las cuatro dimensiones generales del modelo, cuyos niveles de consistencia interna, evaluada con alfa de Cronbach, fueron los siguientes: Autotrascendencia $(\alpha=0,72)$, Conservación $(\alpha=0,62)$, Autopromoción $(\alpha=0,72)$ y Apertura al Cambio $(\alpha=0,70)$.

\section{Escala de Aprecio por Prácticas Sostenibles (APS)}

Esta escala mide un conjunto de actitudes hacia la resolución de problemas socioambientales, a través de una actividad emprendedora equilibrada y respetuosa del entorno. La escala fue desarrollada por los investigadores del estudio, presenta 21 ítems con formato de respuesta tipo Likert del 1 al 5, donde $1=$ totalmente en desacuerdo y $5=$ totalmente de acuerdo, respecto a afirmaciones sobre la preferencia por prácticas con fines sociales y de sostenibilidad. Para analizar la estructura de la escala, se procesó un análisis factorial exploratorio, a través del método de extracción de componentes principales y con una rotación ortogonal Varimax. De esta forma, se obtuvo una óptima medida de adecuación muestral KaiserMeyer-Olkin (KMO; Kaiser, 1974), la cual fue de 0,946 y la prueba de esfericidad de Bartlett resultó significativa, $\chi^{2}(210, n=333)=2614,97, p<0,001$. Los resultados muestran que los 21 ítems están distribuidos en tres factores con eigenvalues mayores que 1. Sin embargo, la interpretación semántica de cada uno de estos factores no aportaba de manera sustantiva a la comprensión del APS, por lo que se decidió observar el gráfico de sedimentación obtenido de la prueba estadística como un criterio complementario para resolver si se utilizarían una o más dimensiones de APS (véase la Figura 1). Finalmente, sumando este criterio a la interpretación de las dimensiones, se optó por trabajar con una dimensión general de APS, la cual explica un 45,04\% de la varianza, presenta cargas factoriales de los ítems que van desde 0,45 hasta 0,80 (ver Tabla 2) y presenta un buen nivel de consistencia interna, evaluada con alfa de Cronbach, de $\alpha=0,96$, lo que es consistente con los resultados obtenidos en otros estudios que hacen uso de la escala (Vera Ruiz, 2019). A mayor puntuación en la escala, mayor es el APS.

Figura 1

Gráfico de Sedimentación del Análisis Factorial Exploratorio de la Escala de APS

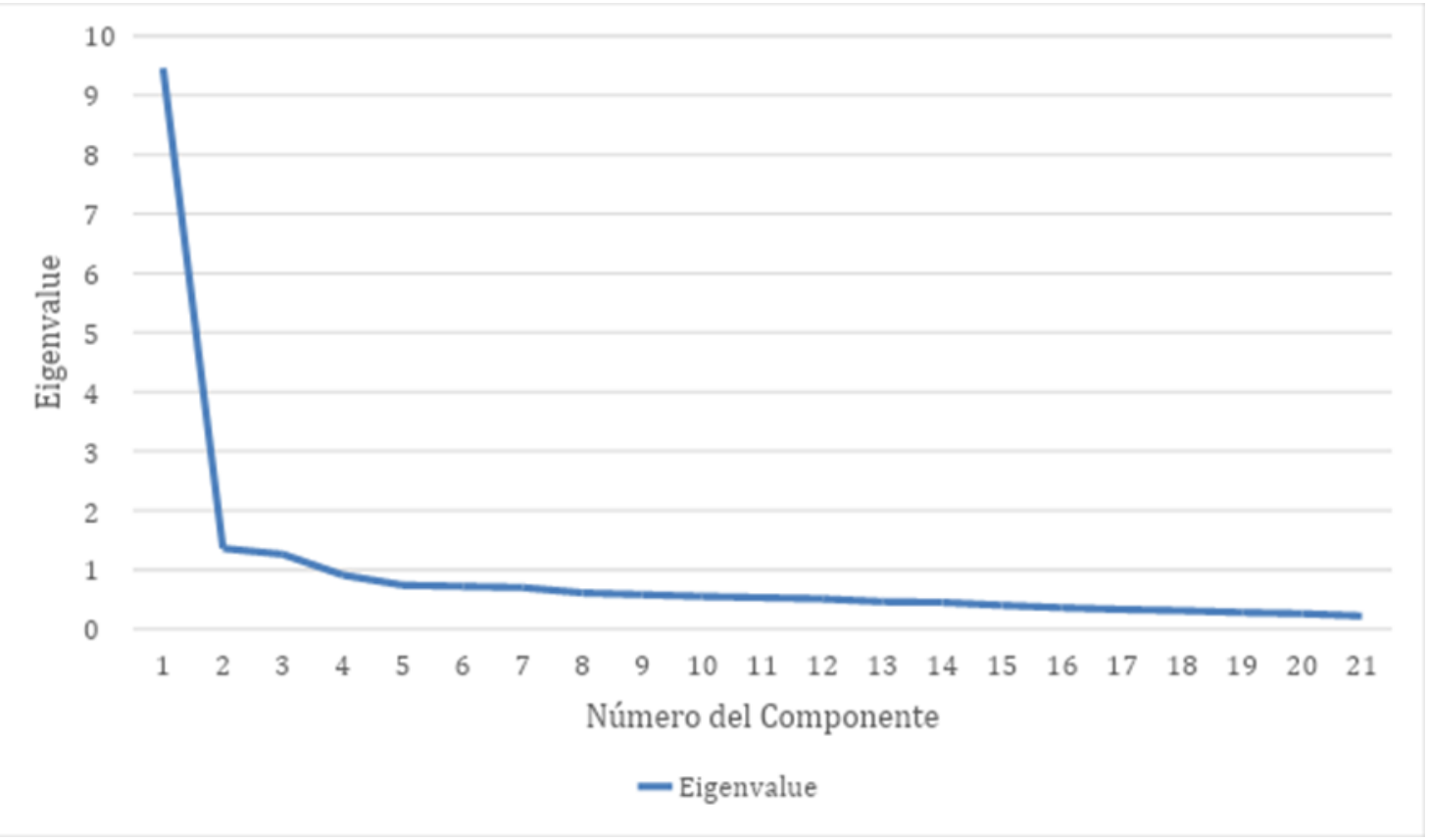




\section{Procedimiento}

La información fue recolectada durante los meses de enero a abril del año 2016, mediante dos procedimientos: (a) presencial, visitando, previo contacto, los locales de los emprendedores y (b) virtual, a través de un correo electrónico, solicitando responder un formulario en Google Docs. En el segundo procedimiento, considerado válido por la literatura (Lyons et al., 2005), se incluyó al inicio del formulario una definición genérica de qué es ser emprendedor, asegurando, así que los participantes efectivamente se identificaran como emprendedores antes de responder. Se usaron formularios idénticos para ambos grupos de empresarios, que permitieron ordenar las respuestas de manera anónima y separada por tipo de emprendimiento. La previa clasificación en los dos grupos no les fue revelada a los participantes para no inducir sesgos en sus respuestas por deseabilidad social. Tanto en la modalidad de aplicación presencial como virtual se presentó a todos los participantes los términos del consentimiento informado, dando cuenta del anonimato, de su participación voluntaria y del manejo ético, riguroso y solo con fines académicos de la información que brindaran.

Tabla 2

Estructura Factorial de la Escala de Aprecio por Prácticas Sostenibles

\begin{tabular}{|c|c|}
\hline Ítem & $\begin{array}{c}\text { Carga } \\
\text { factorial }\end{array}$ \\
\hline Entiendo que para el desarrollo de mi emprendimiento se requiere involucrar de manera progresiva a la comunidad. & 0,631 \\
\hline Me motiva conseguir recursos económicos permanentes para, desde mi emprendimiento, lograr cambios en la sociedad. & 0,679 \\
\hline Entiendo y respeto la diversidad cultural como un elemento que enriquece la labor de mi emprendimiento. & 0,582 \\
\hline Considero importante que el consumidor adquiera productos y/o servicios que promuevan un impacto social positivo. & 0,613 \\
\hline Me interesa que desde mi emprendimiento se promuevan instrumentos de gestión y protección ambiental. & 0,654 \\
\hline Me interesa que mi emprendimiento facilite la generación de políticas públicas para el bien de la comunidad. & 0,686 \\
\hline Desde mi emprendimiento intento conscientizar a las personas sobre las consecuencias de sus prácticas de consumo. & 0,613 \\
\hline Considero que cuando el consumidor está bien informado es posible hacer un comercio más justo. & 0,446 \\
\hline Me satisface actuar desde mi emprendimiento para promover el cuidado de los recursos naturales. & 0,684 \\
\hline Busco asociarme y cooperar con otras organizaciones que tienen los mismos intereses que la mía. & 0,626 \\
\hline Antes que el beneficio económico, me interesa desarrollar capacidades en mis colaboradores y mis clientes. & 0,648 \\
\hline Cuando identifico situaciones sociales que considero injustas, busco cambiarlas desde mi emprendimiento. & 0,753 \\
\hline Mi emprendimiento interactúa con la comunidad, comprende sus problemas y le da soluciones. & 0,709 \\
\hline Busco desarrollar mis productos y servicios pensando en su impacto social y ambiental. & 0,740 \\
\hline Reconozco la realidad social de mi país y eso ha despertado mi sensibilidad social. & 0,771 \\
\hline Mi emprendimiento solo es posible porque trabajo en red con otras personas que comparten mi visión de la sociedad. & 0,580 \\
\hline El interés de mi emprendimiento es construir una sociedad más justa y solidaria. & 0,800 \\
\hline Mi realización personal viene acompañada del crecimiento de mis conciudadanos. & 0,703 \\
\hline Estoy dispuesto a hacer sacrificios económicos por mantener la integridad y misión de mi emprendimiento. & 0,628 \\
\hline Mi historia personal y familiar me ha hecho consciente de mi deber para mejorar la sociedad. & 0,728 \\
\hline Me siento satisfecho de pensar que mi emprendimiento va más allá de mis intereses personales. & 0,727 \\
\hline
\end{tabular}

\section{Análisis de Datos}

En base a los objetivos de investigación propuestos, se desarrollaron análisis descriptivos (media y desviación estándar), análisis de correlaciones lineales de Pearson y análisis de regresión lineal múltiple, para establecer la magnitud y sentido de las relaciones entre los valores, la personalidad y el APS. Asimismo, se llevaron a cabo regresiones logísticas binarias para establecer la capacidad clasificatoria del 
APS, en comparación con los valores y la personalidad. Con esta finalidad se elaboró una variable nominal dicotómica que diferencia a los emprendedores sociales $=0$, de $\operatorname{los}$ comerciales $=1$, bajo los criterios descritos en el acápite de Participantes. Los datos obtenidos fueron procesados con el paquete estadístico IBM SPSS versión 24.

Atendiendo al primer objetivo del estudio, se procesó un análisis de regresión lineal múltiple con el método por pasos, introduciendo como variables predictoras las cuatro dimensiones de valores (Autotrascendencia, Autopromoción, Conservación y Apertura al Cambio) y las cinco dimensiones de la personalidad (Extraversión, Agradabilidad, Apertura Intelectual, Consciencia y Neuroticismo); como variable criterio se usó el indicador de APS.

Para atender al segundo objetivo del estudio, se procesaron 11 regresiones logísticas binarias para clasificar por tipo de emprendimiento a los participantes del estudio. Se optó por el uso de esta técnica estadística de clasificación, porque en la mayoría de los casos las variables predictoras no cumplían con los supuestos de normalidad multivariada y homocedasticidad para la aplicación de técnicas estadísticas de clasificación alternativas (véase Peng et al., 2002).

Para evaluar la significación del modelo se utilizó la prueba de Ómnibus y para determinar la bondad de ajuste del modelo a los datos, la prueba de Hosmer-Lemeshow. En el primero, $p$ debe ser menor a 0,05 y en el segundo, mayor a 0,05. Para estimar el porcentaje de varianza de la variable dependiente explicado por las variables predictoras se utilizaron los coeficientes de determinación $R^{2}$ de Cox y Snell y $R^{2}$ de Nagelkerke. El primero fluctúa entre 0 y 1, pero en la práctica no llega a 1 . El $R^{2}$ de Nagelkerke es una transformación de $R^{2}$ de Cox y Snell, corrigiendo la escala del estadístico para cubrir el rango completo de 0 a 1. La significación estadística de los coeficientes individuales de regresión fue probada usando el estadístico $\chi^{2}$ de Wald.

El primer análisis de regresión logística introdujo como variable predictora el APS y como variable de agrupación, el tipo de emprendimiento de los participantes $(0=$ social versus $1=$ comercial $)$. El segundo análisis de regresión logística se procesó introduciendo las cuatro dimensiones de valores y las cinco dimensiones de personalidad como variables predictoras y el tipo de emprendimiento como variable de agrupación. Las nueve regresiones restantes fueron procesadas introduciendo cada una de las dimensiones de valores o personalidad como variables predictoras, por separado, y el tipo de emprendimiento como variable de agrupación en todos los casos.

\section{Resultados}

\section{Descriptivos y Correlaciones entre APS, Valores y Personalidad}

A nivel descriptivo, considerando el punto medio de las escalas de respuesta en los distintos indicadores evaluados, se puede establecer que la muestra en general reporta altos niveles de APS (valor medio de la escala $=3$ ). Asimismo, con relación a los valores (valor medio de la escala = 3,5 para todas las dimensiones), se aprecia en la muestra un perfil con altos niveles de Autotrascendencia y Apertura al Cambio, así como un nivel medio-alto de Conservación y un nivel medio de Autopromoción. Por su parte, considerando los rasgos de personalidad (valor medio de la escala $=4$ para todas las dimensiones), se observa que la muestra se caracteriza por tener niveles altos de Apertura Intelectual y Consciencia y medio-altos de Agradabilidad y Extraversión. También se observa un nivel bajo de Neuroticismo (ver Tabla 3).

A nivel correlacional, la Tabla 3 muestra asociaciones del APS con algunas dimensiones de valores y personalidad. De manera específica, el APS se correlaciona directamente con los valores de Autotrascendencia y con los rasgos de Agradabilidad, Apertura Intelectual y Extraversión.

En cuanto a los rasgos de personalidad, la Agradabilidad se correlaciona directamente y con un efecto medio con los valores de Autotrascendencia, y de manera inversa y de efecto bajo con los valores de Autopromoción. En ese tenor, el rasgo de Consciencia tiene una relación directa de efecto medio con los valores de Conservación y de efecto bajo con los valores de Autotrascendencia. Asimismo, el rasgo de Extraversión muestra una relación directa de efecto medio con Apertura al Cambio y de bajo efecto con Autotrascendencia y Autopromoción. También, el rasgo de Apertura Intelectual revela una relación directa de efecto bajo con los valores de Autotrascendencia y Apertura al Cambio. 
Tabla 3

Estadísticos Descriptivos y Correlaciones de Pearson entre APS, Valores y Personalidad

\begin{tabular}{|c|c|c|c|c|c|c|c|c|c|c|c|}
\hline Variable & $M(D E)$ & 1 & 2 & 3 & 4 & 5 & 6 & 7 & 8 & 9 & 10 \\
\hline 1. APS & $4,21(0,59)$ & - & & & & & & & & & \\
\hline \multicolumn{12}{|l|}{ Valores } \\
\hline 2. Autotrascendencia & $5,32(0,55)$ & $0,36^{* *}$ & - & & & & & & & & \\
\hline 3. Autopromoción & $3,71(1,01)$ & $-0,09$ & 0,01 & - & & & & & & & \\
\hline 4. Conservación & $4,30(0,77)$ & $-0,02$ & $0,28^{* *}$ & $0,44^{* *}$ & - & & & & & & \\
\hline 5. Apertura al cambio & $4,70(0,71)$ & 0,08 & $0,34^{* *}$ & $0,33^{* *}$ & $0,16^{* *}$ & - & & & & & \\
\hline \multicolumn{12}{|l|}{ Personalidad } \\
\hline 6. Extraversión & $5,02(0,78)$ & $0,15^{* *}$ & $0,13^{*}$ & $0,12^{*}$ & $-0,07$ & $0,29 * *$ & - & & & & \\
\hline 7. Agradabilidad & $5,05(0,62)$ & $0,29 * *$ & $0,28^{* *}$ & $-0,18^{* *}$ & $-0,07$ & 0,02 & $0,34^{* *}$ & - & & & \\
\hline 8. Consciencia & $5,31(0,64)$ & 0,09 & $0,12^{*}$ & 0,01 & $0,26^{* *}$ & $-0,03$ & $0,33^{* *}$ & $0,37^{* *}$ & - & & \\
\hline 9. Neuroticismo & $3,29(0,68)$ & $-0,08$ & $-0,07$ & 0,10 & 0,01 & $-0,00$ & $-0,34^{* *}$ & $-0,39^{* *}$ & $-0,36^{* *}$ & - & \\
\hline 10. Apertura intelectual & $5,39(0,69)$ & $0,21^{* *}$ & $0,13^{*}$ & 0,03 & $-0,14^{*}$ & $0,19^{* *}$ & $0,57^{* *}$ & $0,32^{* *}$ & $0,41^{* *}$ & $-0,34^{* *}$ & - \\
\hline
\end{tabular}

Nota. $n=333 ;{ }^{*} p<0,05 ; * * p<0,01$.

El análisis de regresión lineal múltiple arrojó tres pasos, en el que el último modelo identificado explica un 19\% de la varianza del APS. Las variables predictoras del APS serían los valores de Autotrascendencia, con un tamaño del efecto grande, y los rasgos de personalidad de Agradabilidad y Apertura Intelectual, con tamaños del efecto pequeños (véase Richard et al., 2003; ver Tabla 4). Con relación al carácter predictivo de los valores, estos resultados sustentan la hipótesis 1, en la que se enunciaba un efecto directo de la Autotrascendencia en el APS. Sin embargo, se desestiman las hipótesis 2, 3 y 4, pues los demás valores no se relacionan ni predicen el APS. En cuanto a los rasgos de personalidad, los resultados sustentan las hipótesis 5 y 6 del estudio, que daban cuenta de sendas relaciones predictivas de influencia de la Agradabilidad y la Apertura Intelectual en el APS. Asimismo, se sustenta la hipótesis 7 del estudio que establecía que los rasgos de Consciencia, Extraversión y Neuroticismo no predecirían el APS. Cabe mencionar de manera específica que la baja consistencia interna del indicador de Neuroticismo podría haber afectado las relaciones de esta variable con las otras variables del estudio.

Tabla 4

Regresión Lineal Múltiple por Pasos de Valores y Personalidad en APS

\begin{tabular}{|c|c|c|c|c|c|c|c|c|c|}
\hline Variable & $B$ & B & $R^{2}$ & $\Delta R^{2}$ & $F$ & $g l 1$ & $g l 2$ & $p$ & $95 \%$ IC de $B$ \\
\hline Paso 1 & & & 0,136 & 0,136 & 52,29 & 1 & 331 & $<0,001$ & \\
\hline Autotrascendencia & 0,395 & 0,369 & & & & & & 0,001 & {$[0,288,0,503]$} \\
\hline Paso 2 & & & 0,175 & 0,039 & 35,01 & 2 & 330 & $<0,001$ & \\
\hline Autotrascendencia & 0,333 & 0,311 & & & & & & 0,001 & {$[0,223,0,443]$} \\
\hline Agradabilidad & 0,194 & 0,205 & & & & & & 0,001 & {$[0,097,0,291]$} \\
\hline Paso 3 & & & 0,189 & 0,014 & 25,50 & 3 & 329 & $<0,001$ & \\
\hline Autotrascendencia & 0,326 & 0,305 & & & & & & 0,001 & {$[0,217,0,425]$} \\
\hline Agradabilidad & 0,159 & 0,168 & & & & & & 0,010 & {$[0,058,0,259]$} \\
\hline Apertura intelectual & 0,105 & 0,123 & & & & & & 0,050 & {$[0,017,0,193]$} \\
\hline
\end{tabular}

Nota. $B=$ beta no estandarizado; $B=$ beta estandarizado; $\Delta R^{2}=$ cambio en $R^{2}$. 


\section{Análisis de Regresión Logística Binaria del APS, los Valores y la Personalidad para la Clasificación de Emprendedores según Tipo de Emprendimiento (Social Versus Comercial)}

El primer análisis arroja una función logística binaria significativa, prueba de Ómnibus $\chi^{2}(1, n=333)=$ $55,943, p<0,001$. El modelo también muestra un buen ajuste a los datos: prueba de Hosmer-Lemeshow $\chi^{2}(8, n$ $=333)=9,857, p=0,275$. Dos medidas adicionales de bondad de ajuste del modelo son la $R^{2}$ de Cox y Snell $=$ 0,155 y $R^{2}$ de Nagelkerke $=0,209$, que sugieren que la APS explica entre un $16 \%$ y un $21 \%$ de la varianza de la clasificación de emprendedores.

La Tabla 5 muestra los coeficientes de la regresión logística binaria que buscan evaluar el efecto de la APS en la clasificación por tipo de emprendimiento. Los resultados muestran que la puntuación en APS diferencia a los emprendedores sociales de los comerciales, siendo sus niveles más altos en el primer grupo, lo que sustenta lo propuesto en la hipótesis 8. Asimismo, los coeficientes de la función de clasificación del APS por tipo de emprendimiento obtenidos con la presente muestra permiten asignar correctamente al $68,8 \%$ de los participantes del estudio.

\section{Tabla 5}

Coeficientes No Estandarizados de la Función Clasificatoria del APS sobre el Tipo de Emprendimiento

\begin{tabular}{lccccrr}
\hline & $B$ & Error Estándar $B$ & $\chi^{2}$ de Wald & $p$ & $\begin{array}{c}\text { Odd ratio } \\
(\text { OR })\end{array}$ & 95\% IC de OR \\
\hline Constante & 7,769 & 1,143 & 46,178 & 0,001 & 2365,218 & \\
APS & $-1,726$ & 0,262 & 43,386 & 0,001 & 0,178 & {$[0,106,0,297]$} \\
\hline
\end{tabular}

Una segunda regresión logística binaria, introduciendo las cuatro dimensiones de valores y las cinco dimensiones de personalidad como variables predictoras y el tipo de emprendimiento como variable agrupación, arroja una función logística significativa, prueba de Ómnibus $\chi^{2}(9, n=333)=126,309, p<$ 0,001. Los resultados del modelo también muestran un buen ajuste de los datos al mismo: prueba de Hosmer-Lemeshow $\chi^{2}(8, n=333)=13,059, p=0,110$. Los coeficientes de determinación $R^{2}$ de Cox y Snell $=0,316$ y $R^{2}$ de Nagelkerke $=0,426$ muestran que en conjunto las cinco dimensiones de personalidad y las cuatro dimensiones de valores utilizadas como variables predictoras explican entre un $32 \%$ y un $43 \%$ de la varianza de la clasificación de emprendedores.

La significación estadística de los coeficientes individuales de regresión se muestra en la Tabla 6. En cuanto a las dimensiones de personalidad, las que diferencian a emprendedores sociales de comerciales son Agradabilidad, Consciencia y AperturaIntelectual, mientras que, en el caso de los valores, las dimensiones que diferencian a emprendedores sociales de comerciales son Autotrascendencia, Autopromoción y Conservación. En conjunto, la personalidad y los valores permiten clasificar apropiadamente al 76\% de los participantes de esta muestra.

Esto último sugiere que, en conjunto, los valores y la personalidad actúan como mejores predictores del tipo de emprendimiento que el APS, desestimando la hipótesis 9 del estudio. Sin embargo, los análisis de regresión logística binaria restantes, introduciendo cada una de las dimensiones de valores o personalidad por separado, sugieren que estas dimensiones por sí solas no clasifican la pertenencia de los participantes a su respectivo tipo de emprendimiento mejor que el APS.

En la Tabla 7 se reportan los porcentajes de clasificación correcta de cada uno de los indicadores de valores y personalidad, según tipo de emprendimiento. 
Tabla 6

Coeficientes No Estandarizados de la Función Clasificatoria de los Valores y la Personalidad sobre el Tipo de Emprendimiento

\begin{tabular}{lccrcrc}
\hline \multicolumn{1}{c}{ Variable } & $B$ & Error Estándar $B$ & $\chi^{2}$ de Wald & $p$ & Odd ratio (OR) & $95 \%$ IC de OR \\
\hline Constante & 8,078 & 2,575 & 9,843 & 0,002 & 3224,145 & \\
Autotrascendencia & $-1,522$ & 0,353 & 18,640 & 0,001 & 0,218 & {$[0,109,0,436]$} \\
Autopromoción & 0,420 & 0,173 & 5,883 & 0,050 & 1,523 & {$[1,084,2,139]$} \\
Conservación & 0,797 & 0,254 & 9,801 & 0,010 & 2,218 & {$[1,347,3,653]$} \\
Apertura al cambio & $-0,204$ & 0,231 & 0,786 & 0,375 & 0,815 & {$[0,519,1,281]$} \\
Extraversión & $-0,030$ & 0,231 & 0,017 & 0,897 & 0,970 & {$[0,619,1,526]$} \\
Agradabilidad & $-0,693$ & 0,283 & 5,979 & 0,050 & 0,500 & {$[0,287,0,872]$} \\
Consciencia & 1,077 & 0,307 & 12,313 & 0,001 & 2,935 & {$[1,609,5,355]$} \\
Neuroticismo & 0,123 & 0,237 & 0,268 & 0,604 & 1,131 & {$[0,710,1,800]$} \\
Apertura intelectual & $-1,102$ & 0,293 & 14,135 & 0,001 & 0,332 & {$[0,187,0,597]$} \\
\hline
\end{tabular}

Tabla 7

Porcentaje de Casos Clasificados Correctamente al Analizar por Separado los Valores o Personalidad

\begin{tabular}{lc}
\hline \multicolumn{1}{c}{ Variable } & Porcentaje de clasificación correcta \\
\hline Autotrascendencia & 64,0 \\
Autopromoción & 64,9 \\
Conservación & 68,8 \\
Apertura al cambio & 61,0 \\
Extraversión & 58,3 \\
Agradabilidad & 64,9 \\
Consciencia & 58,9 \\
Neuroticismo & 58,9 \\
Apertura intelectual & 65,5 \\
\hline
\end{tabular}

\section{Discusión}

A nivel descriptivo, los resultados sugieren que la muestra total del presente estudio está caracterizada por un elevado nivel de APS, siendo este mayor en los emprendedores sociales que en los comerciales. Además, de acuerdo con lo hipotetizado, el APS como medida clasifica adecuadamente a los miembros de estos dos grupos de emprendedores. Se puede concluir, entonces, que se trata de un constructo que, acompañado de una buena medición, podría ser un adecuado predictor del tipo de emprendimiento que una persona podría llegar a desarrollar.

Si bien, contra lo hipotetizado, la capacidad clasificatoria del APS es menor que la de la totalidad de dimensiones de valores y personalidad, pues en conjunto ambos constructos han conseguido clasificar con mayor precisión a los participantes del estudio, es importante señalar que en este proceso se han requerido utilizar nueve dimensiones (cuatro de valores y cinco de personalidad) versus una en el APS. Asimismo, los resultados muestran que las dimensiones de los valores y la personalidad por separado no dan cuenta de un mejor funcionamiento clasificatorio que el APS. Así, el APS sería una medida clasificatoria más parsimoniosa cuando se compara con dimensiones específicas de valores o personalidad por separado. 
Como había sido hipotetizado, el funcionamiento estadístico observado confirma la capacidad predictiva directa que los valores de autotrascendencia y los rasgos de agradabilidad y apertura intelectual tienen sobre el APS. Esto es consistente con los resultados de estudios previos, en los que los dos rasgos de personalidad mencionados se relacionaban directamente con el APS (Caballero et al., 2014; Nga \& Shamuganathan, 2010). Vale señalar que las tres variables mencionadas son predominantes en los emprendedores sociales y cumplen una adecuada función clasificatoria en los miembros de este grupo de participantes. A nivel conceptual, estos resultados son compatibles con la caracterización que se hace en la literatura sobre el comportamiento emprendedor de orientación social, el cual, desde una visión sistémica e innovadora, busca el bien común y el cuidado de otros, bajo estrategias de creación de valor que armonizan resultados sociales, económicos y ambientales (Austin et al., 2006; Kuckertz \& Wagner, 2010; Mair \& Martí, 2006). Los valores de autotrascendencia y los rasgos de agradabilidad son promotores de comportamientos prosociales (Hardy et al., 2010; Lönnqvist et al., 2013) orientados a satisfacer la necesidad de apoyo a otros (Auné et al., 2014), lo que sería congruente con la disposición de servicio y posicionamiento moral que implica el APS, al cual se suma la apertura intelectual, fundamental para conseguir los objetivos del emprendimiento desde una perspectiva innovadora.

De esta forma, el presente estudio también aporta evidencia empírica para nutrir el debate académico sobre los cuestionados fines instrumentales del emprendimiento social y su verosimilitud ética (Chell et al., 2016). Este debate viene problematizando el utilitarismo del fenómeno para disfrazar de buenas intenciones algunos objetivos emprendedores meramente individualistas y monetarios (Adams et al., 2019; Bröckling, 2007/2015). La lectura de los resultados muestra las diferencias entre los grupos de emprendedores sociales y comerciales, siendo los primeros más afables, creativos y autotrascendentes que los segundos y, por ende, mayormente orientados hacia el APS. Por su parte, los emprendedores comerciales se presentan más orientados por valores de autopromoción y conservación, así como por el rasgo de consciencia. Este hecho plantea que, en efecto, hay características axiológicas, de personalidad y actitudinales que diferencian a los emprendedores sociales de los comerciales y, por lo tanto, ratificarían la genuina intención de los primeros por cuidar del bienestar de las personas y del medio ambiente, posicionándose como actores determinados a mitigar la pobreza y lograr el cambio institucional (Saebi et al., 2019). En cuanto a los emprendedores comerciales participantes, se observan características afines con la convencional meticulosidad y competitividad del perfil emprendedor clásico (Brandstätter, 2011; Marulanda Valencia et al., 2014), pero difieren en tener altos valores de conservación, lo que podría entenderse bajo el reconocimiento del contexto colectivista y conservador peruano, donde se busca mantener el orden social (Espinosa Pezzia et al., 2017). Lo anterior resulta coherente con la consideración de que las condiciones del contexto refuerzan o inhiben ciertas características psicosociales de los emprendedores (Brandstätter, 2011; Zhao \& Seibert, 2006), lo que intensifica la idea de que son los valores de autotrascendencia y los rasgos de apertura y agradabilidad los que favorecen una mejor disposición hacia la sostenibilidad. Por lo tanto, estos valores pueden ser aspectos a incluir en las estrategias de promoción de prácticas emprendedoras locales de orientación social. Al respecto, estudios previos en el Perú proponen el APS como un elemento actitudinal determinante en la conformación de emprendimientos sociales (Vera Ruiz et al., 2019, 2020).

Lo señalado adquiere particular importancia en el contexto de un país conservador, colectivista y materialista como el Perú (Castaman Díaz, 2017; Cueto et al., 2017), en el que la actividad emprendedora, en la mayoría de los casos, se inicia predominantemente como medio para cubrir las necesidades básicas de las personas y su permanencia en el tiempo es muy corta (Serida et al., 2010). Dado que en la literatura se observa que es justamente en economías con mayores deficiencias y brechas sociales donde el emprendimiento social ha tomado más vuelo (Austin et al., 2006; Bikse et al., 2015; Bosma et al., 2015), la condición de consciencia y disposición a la sostenibilidad que implica el APS, como atributo presente en los emprendedores peruanos, resultaría un recurso psicosocial con potencial para promover y consolidar la permanencia de múltiples emprendimientos, cuya formación ha sido originalmente motivada por la supervivencia, redirigiéndolos hacia una actividad emprendedora que genera valor económico, social y ambiental. Se trataría, entonces, de deconstruir la condición de precariedad y supervivencia del emprendimiento local, hacia una lógica de sostenibilidad consistente con el concepto del APS.

En cuanto a las implicancias prácticas y sociales del estudio, es pertinente analizar las características psicosociales de los individuos, considerando que ellos, en tanto actores productivos inmersos y condicionados por un sistema de mercado complejo e interconectado, tienen incidencia directa en los procesos productivos del mismo. Por ello, desarrollar un instrumento sobre el APS suma al campo de la 
comprensión del fenómeno emprendedor, desde una medición individual de la disposición a la sostenibilidad, ofreciendo la posibilidad de evaluar niveles de orientación social en diferentes tipos de emprendedores.

El valor de este trabajo se basa en el análisis de las relaciones, novedosas y contextualizadas, entre variables psicosociales que permiten enriquecer la comprensión del fenómeno del emprendimiento. El análisis de características axiológicas, de personalidad y actitudinales en el ámbito de estudio del emprendimiento local pone en evidencia dimensiones de análisis más inclusivas, solidarias y consistentes que las tradicionalmente empleadas, las que se han centrado en la búsqueda de independencia, logro y lucro como un ideal del emprendimiento (Marulanda Valencia et al., 2014). Esto se basa en la idea de que el APS, al encontrarse relacionado con aquellos valores y rasgos de personalidad más sensibles a ser modificados por la influencia sociocultural o por coyunturas críticas (véase Fischer \& Boer, 2015; Manfredo et al., 2017; Parks-Leduc et al., 2015), es una disposición actitudinal con el potencial de ser transformada, en aras de una actividad emprendedora sostenible y solidaria (Vera Ruiz et al., 2020). Esto es viable en la medida en que el encuentro y la convivencia con la diversidad social y cultural, así como el reconocimiento de la escasez de recursos naturales, reforzarían posicionamientos universalistas y prosociales (Schwartz, 2010) consistentes con la promoción del APS en el campo de la investigación sobre el emprendimiento en contextos precarios y excluyentes como el peruano.

Finalmente, en consonancia con lo anterior, se reconoce como limitación de este estudio la novedad del tema en el contexto local y el reducido acceso a emprendedores sociales, sobre todo porque dentro de esta denominación existe una diversidad de modalidades e hibridaciones que no hacen sencillo reconocerlos como tales (Saebi et al., 2019). La estrategia metodológica de selección de participantes se basó en rigurosos criterios para identificar a este grupo, pero ello pudo haber dejado fuera a emprendedores que, sin estar explícitamente registrados en entidades del ecosistema emprendedor social local, podrían ejercer prácticas consecuentes con una orientación sostenible.

\section{Referencias}

Abhi, S. (2017). Can social entrepreneurs do well by doing good? Blending social and economic value creation — An investigation. Academy of Entrepreneurship Journal, 23(2), Artículo 3. https://www.abacademies.org/articles/can-social-entrepreneurs-do-wellby-doing-good--blending-social-and-economic-value-creation--an-investigation-6671.html

Abu-Saifan, S. (2012). Social entrepreneurship: Definition and boundaries. Technology Innovation Management Review, 2(February 2012), 22-27. https://doi.org/10.22215/timreview/523

Adams, G., Estrada-Villalta, S., Sullivan, D. \& Markus, H. R. (2019). The psychology of neoliberalism and the neoliberalism of psychology. Journal of Social Issues, 75(1). 189-216. https://doi.org/10.1111/josi.12305

Antoncic, B., Bratkovic Kregar, T., Singh, G. \& DeNoble, A. F. (2015). The big five personality-entrepreneurship relationship: Evidence from Slovenia. Journal of Small Business Management, 53(3), 819-841. https://doi.org/10.1111/jsbm.12089

Auné, S. E., Blum, D., Abal, F. J. P., Lozzia, G. S. \& Attorresi, H. F. (2014). La conducta prosocial: estado actual de la investigación. Perspectivas en Psicología, 11(2), 21-33. http://www.redalyc.org/articulo.oa?id=483547666003

Austin, J., Stevenson, H. \& Wei-Skillern, J. (2006). Social and commercial entrepreneurship: Same, different, or both? Entrepreneurship Theory and Practice, 30(1), 1-22. https://doi.org/10.1111/i.1540-6520.2006.00107.x

Beramendi, M., Espinosa, A. \& Ara, S. (2013). Perfiles axiológicos de estudiantes de tres carreras universitarias: funciones discriminantes de tres lecturas de la teoría de Schwartz. LIBERABIT: Revista Peruana de Psicología, 19(1), 45-54. http://www.scielo.org.pe/pdf/liber/v19n1/a05v19n1.pdf

Bikse, V., Rivza, B. \& Riemere, I. (2015). The social entrepreneur as a promoter of social advancement. Procedia: Social and Behavioral Sciences, 185, 469-478. https://doi.org/10.1016/j.sbspro.2015.03.405

Bosma, N., Schøtt, T., Terjesen, S. \& Kew, P. (2015). Social entrepreneurship (Special topic report). Global Entrepreneurship Monitor. https://www.gemconsortium.org/file/open?fileId=49542

Brandsen, T. \& Karré, P. M. (2011). Hybrid organizations: No cause for concern? Journal of Public Administration, 34(13), 827-836. https://doi.org/10.1080/01900692.2011.605090

Brandstätter, H. (2011). Personality aspects of entrepreneurship: A look at five meta-analyses. Personality and Individual Differences, 51(3), 222-230. https://doi.org/10.1016/j.paid.2010.07.007

Bröckling, U. (2015). El self emprendedor: sociología de una forma de subjetivación (K. Böhmer, Trad.). Editorial Universidad Alberto Hurtado. (Obra original publicada en 2007)

Bruyat, C. \& Julien, P. -A. (2001). Defining the field of research in entrepreneurship. Journal of Business Venturing, 16(2), 165-180. https://doi.org/10.1016/S0883-9026(99)00043-9

Caballero, S., Fuchs, R. M., Prialé, M. A. \& Nga, J. K. H. (2014). The influence of the big 5 personality traits on the social enterprise start-up intentions: A Peruvian case. Taylor's Business Review, 4(1), 87-107. http://emes.net/content/uploads/publications/the-influence-ofpersonality-traits-on-social-enterprise-start-up-the-case-of-peruvian-social-entrepreneurs/caballero_et_al. ecps-lg13-07 eng.pdf

Calderón Prada, A. (2003). Satisfacción marital desde la perspectiva de la psicología evolutiva en parejas urbano marginales de Lima [Tesis de licenciatura no publicada]. Pontificia Universidad Católica del Perú.

Cartwright, W. \& Craig, J. L. (2006). Sustainability: Aligning corporate governance, strategy and operations with the planet. Business Process Management Journal, 12(6), 741-750. https://doi.org/10.1108/14637150610710909 
Carver, C. S. \& Scheier, M. F. (1981). Attention and self-regulation: A control-theory approach to human behavior. Springer-Verlag. https://doi.org/10.1007/978-1-4612-5887-2

Castaman Díaz, K. (2017). Tradición y postmaterialismo en el Perú (1996-2012): análisis a partir de la Encuesta Mundial de Valores (Cuadernos de Investigación $\mathrm{N}^{\circ}$ 14). Pontificia Universidad Católica del Perú, Instituto de Opinión Pública. http://repositorio.pucp.edu.pe/index/handle/123456789/69769

Cater III, J. J., Collins, L. A. \& Beal, B. D. (2017). Ethics, faith, and profit: Exploring the motives of the U.S. fair trade social entrepreneurs. Journal of Business Ethics, 146(1), 185-201. https://doi.org/10.1007/s10551-015-2934-4

Chell, E. (2008). The entrepreneurial personality: A social construction. Second edition. Routledge. https://doi.org/10.4324/9780203938638

Chell, E., Spence, L. J., Perrini, F. \& Harris, J. D. (2016). Social entrepreneurship and business ethics: Does social equal ethical? Journal of Business Ethics, 133(4), 619-625. https://doi.org/10.1007/s10551-014-2439-6

Cope, J. (2005). Toward a dynamic learning perspective of entrepreneurship. Entrepreneurship Theory and Practice, 29(4), 373-397. https://doi.org/10.1111/j.1540-6520.2005.00090.x

Cueto, R. M., Espinosa, A. \& Robles, R. (2017). Narrativas sobre la sociedad peruana y la identidad nacional en universitarios peruanos. Límite: Revista Interdisciplinaria de Filosofía \& Psicología, 12(38), 7-21. https://www.revistalimite.cl/index.php/limite/article/view/68

Espinosa, A., Herschkowicz, S. \& Genna, K. (2011). Correlatos psicológicos de las intenciones y comportamientos migratorios de jóvenes peruanos de clase media y alta. Psicoperspectivas, 10(1), 99-124. https://doi.org/10.5027/psicoperspectivas-Vol10-Issue1fulltext-143

Espinosa Pezzia, A., Soares Da Silva, A., Contreras Ibáñez, C., Cueto, R. M., García Rengifo, A., Ortolano, F., Valencia, J. \& Vera Ruiz, A. (2017). Identidad nacional y sus relaciones con la ideología y el bienestar en cinco países de América Latina. Avances en Psicología Latinoamericana, 35(2), 351-374. https://doi.org/10.12804/revistas.urosario.edu.co/apl/a.3765

Fischer, R. \& Boer, D. (2015). Motivational basis of personality traits: A meta-analysis of value-personality correlations. Journal of Personality, 83(5), 491-510. https://doi.org/10.1111/jopy.12125

García Anchorena, R., Grimaldo Muchotrigo, M. \& Manzanares Medina, E. L. (2016). Jerarquía de valores entre estudiantes de secundaria de colegio religioso y colegio laico de Lima. LIBERABIT: Revista Peruana de Psicología, 22(2), 229-238. https://doi.org/10.24265/liberabit.2016.v22n2.10

García Villaverde, P. M. \& Valencia de Lara, P. (2009). Factores de éxito del proceso de puesta en marcha de la empresa: valores culturales, redes sociales y ayudas públicas. Investigaciones Europeas de Dirección y Economía de la Empresa, 15(3), 101-117. https://doi.org/10.1016/S1135-2523(12)60103-8

Global Entrepreneurship Research Association. (2017). Global report 2016/17. Babson College, Universiti Tun Abdul Razak, Universidad del Desarrollo \& Tecnológico de Monterrey. http://www.gemconsortium.org/report/49812

Goldberg, L. R. (1990). An alternative "description of personality": The big-five factor structure. Journal of Personality and Social Psychology, 59(6), 1216-1229. https://doi.org/10.1037/0022-3514.59.6.1216

Gurrieri, A. R. (2013). Networking entrepreneurs. The Journal of Socio-Economics, 47, 193-204. https://doi.org/10.1016/j.socec.2013.09.007

Guzmán Vásquez, A. \& Trujillo Dávila, M. A. (2008). Emprendimiento social - revisión de literatura. Estudios Gerenciales, 24(109), 105-125. https://doi.org/10.1016/S0123-5923(08)70055-X

Hardy, S. A., Carlo, G. \& Roesch, S. C. (2010). Links between adolescents' expected parental reactions and prosocial behavioral tendencies: The mediating role of prosocial values. Journal of Youth and Adolescence, 39(1), Article 84. https://doi.org/10.1007/s10964-008-9383-7

Herrera, D. \& Lens, W. (13 al 18 de julio de 2003). Changes in future time perspective according to the educational level and its association with values among adolescents of private and public schools and universities in Lima [Simposio]. 29th Interamerican Congress of Psychology, Lima, Perú.

Imhoff, D. \& Brussino, S. (2013). Estudio exploratorio de las características psicométricas del Portrait Values Questionnaire en el contexto de Córdoba - Argentina. Revista Colombiana de Psicología, 22(1), 135-149. https://repositorio.unal.edu.co/bitstream/handle/unal/33525/20594173294-2-PB.pdf?sequence=3\&isAllowed=y

Inglehart, R. (1981). Post-materialism in an environment of insecurity. American Political Science Review, 75(4), 880-900. https://doi.org/10.2307/1962290

İrengün, O. \& Arıkboğa, Ş. (2015). The effect of personality traits on social entrepreneurship intentions: A field research. Procedia: Social and Behavioral Sciences, 195, 1186-1195. https://doi.org/10.1016/j.sbspro.2015.06.172

Kaiser, H. F. (1974). An index of factorial simplicity. Psychometrika, 39(1), 31-36. https://doi.org/10.1007/BF02291575

Kecharananta, N. \& Baker, H. G. (1999). Capturing entrepreneurial values. Journal of Applied Social Psychology, 29(4), 820-833. https://doi.org/10.1111/j.1559-1816.1999.tb02027.x

Kneiding, C. \& Tracey, P. (2009). Towards a performance measurement framework for community development finance institutions in the UK. Journal of Business Ethics, 86(3), 327-345. https://doi.org/10.1007/s10551-008-9850-9

Kuckertz, A. \& Wagner, M. (2010). The influence of sustainability orientation on entrepreneurial intentions - Investigating the role of business experience. Journal of Business Venturing, 25(5), 524-539. https://doi.org/10.1016/j.jbusvent.2009.09.001

Leadbeater, C. (1997). The rise of the social entrepreneur. Demos.

Leisinger, K. M. (2007). Corporate philanthropy: The "top of the pyramid". Business and Society Review, $112(3), 315-342$. https://doi.org/10.1111/j.1467-8594.2007.00299.x

Levie, J. \& Hart, M. (2011). Business and social entrepreneurs in the UK: Gender, context and commitment. International Journal of Gender and Entrepreneurship, 3(3), 200-217. https://doi.org/10.1108/17566261111169304

Liang, C. -T., Peng, L. -P, Yao, S. -N. \& Liang, C. (2015). Developing a social enterprise performance scale and examining the relationship between entrepreneurs' personality traits and their perceived enterprise performance. Journal of Entrepreneurship, Management and Innovation, 11(3), 89-116. https://doi.org/10.7341/20151135

Lönnqvist, J. -E., Verkasalo, M., Wichardt, P. C. \& Walkowitz, G. (2013). Personal values and prosocial behaviour in strategic interactions: Distinguishing value-expressive from value-ambivalent behaviours. European Journal of Social Psychology, 43(6), 554-569. https://doi.org/10.1002/ejsp.1976

Luca, M. R. (2017). Entrepreneurial personality and entrepreneurial behaviour. Bulletin of the Transilvania University of Braşov. Series VII: Social Sciences and Law, Psychology and Pedagogy, 10(1), 19-28. http://webbut.unitbv.ro/Bulletin/Series\%20VII/2017/BULETIN\%20I\%20PDF/04_Luca.pdf

Lyons, A. C., Cude, B., Lawrence, F. C. \& Gutter, M. (2005). Conducting research online: Challenges facing researchers in family and consumer sciences. Family \& Consumer Sciences Research Journal, 33(4), 341-356. https://doi.org/10.1177/1077727X04274116 
Mair, J. \& Martí, I. (2006). Social entrepreneurship research: A source of explanation, prediction, and delight. Journal of World Business, 41(1), 36-44. https://doi.org/10.1016/j.jwb.2005.09.002

Mair, J. \& Noboa, E. (2006). Social entrepreneurship: How intentions to create a social venture are formed. En J. Mair, J. Robinson \& K. Hockerts (Eds.), Social entrepreneurship (pp. 121-135). Palgrave Macmillan.

Manfredo, M. J., Bruskotter, J. T., Teel, T. L., Fulton, D., Schwartz, S. H., Arlinghaus, R., Oishi, S., Uskul, A. K., Redford, K., Kitayama, S. \& Sullivan, L. (2017). Why social values cannot be changed for the sake of conservation. Conservation Biology, 31(4), 772-780. https://doi.org/10.1111/cobi.12855

Martin, R. L. \& Osberg, S. (2007). Social entrepreneurship: The case for definition. Stanford Social Innovation Review, 5(2), Artículo 34. https://ssir.org/articles/entry/social_entrepreneurship the case for definition

Marulanda Valencia, F. Á., Montoya Restrepo, I. A. \& Vélez Restrepo, J. M. (2014). Teorías motivacionales en el estudio del emprendimiento. Pensamiento \& Gestión, 36, 206-238. https://doi.org/10.14482/pege.36.5571

McCrae, R. R. \& Costa Jr., P. T. (1990), Personality in adulthood. Guilford Press.

McCrae, R. R. \& Costa Jr., P. T. (1992). Discriminant validity of NEO-PIR Facet Scales. Educational and Psychological Measurement, 52(1), 229-237. https://doi.org/10.1177/001316449205200128

Mezulis, A. H., Abramson, L. Y., Hyde, J. S. \& Hankin, B. L. (2004). Is there a universal positivity bias in attributions? A metaanalytic review of individual, developmental, and cultural differences in the self-serving attributional bias. Psychological Bulletin, 130(5), 711-747. https://doi.org/10.1037/0033-2909.130.5.711

Moriano, J. A., Trejo, E. \& Palací, F. J. (2001). El perfil psicosocial del emprendedor: un estudio desde la perspectiva de los valores. Revista de Psicología del Trabajo y de las Organizaciones, 16(2), 229-242. https://doi.org/10.1174/021347401317351152

Morris, C. G. \& Maisto, A. A. (2011). Introducción a la psicología: décimotercera edición. Pearson.

Nga, J. K. H. \& Shamuganathan, G. (2010). The influence of personality traits and demographic factors on social entrepreneurs hip start up intentions. Journal of Business Ethics, 95(2), 259-282. https://.doi.org/10.1007/s10551-009-0358-8

Ormeño Coronado, B. (2014). Emprendimiento femenino: propuesta de un perfil en base al propio discurso de mujeres, desde una perspectiva cualitativa. Revista Gestión de las Personas y Tecnología, 7(19), 20-25. https://www.revistagpt.usach.cl/sites/revistagpt/files/19_pdf interactivocomprimido.pdf

Parks-Leduc, L., Feldman, G. \& Bardi, A. (2015). Personality traits and personal values: A meta-analysis. Personality and Social Psychology Review, 19(1), 3-29. https://doi.org/10.1177/1088868314538548

Peng, C. -Y. J., Lee, K. L. \& Ingersoll, G. M. (2002). An introduction to logistic regression analysis and reporting. The Journal of Educational Research, 96(1), 3-14. https://doi.org/10.1080/00220670209598786

Richard, F. D., Bond Jr., C. F. \& Stokes-Zoota, J. J. (2003). One hundred years of social psychology quantitatively described. Review of General Psychology, 7(4), 331-363. https://doi.org/10.1037/1089-2680.7.4.331

Roccas, S., Sagiv, L., Schwartz, S. H. \& Knafo, A. (2002). The big five personality factors and personal values. Personality and Social Psychology Bulletin, 28(6), 789-801. https://doi.org/10.1177/0146167202289008

Rokeach, M. (1973). The nature of human values. Free Press.

Ruskin, J., Seymour, R. G. \& Webster, C. M. (2016). Why create value for others? An exploration of social entrepreneurial motives. Journal of Small Business Management, 54(4), 1015-1037. https://doi.org/10.1111/jsbm.12229

Saebi, T., Foss, N. J. \& Linder, S. (2019). Social entrepreneurship research: Past achievements and future promises. Journal of Management, 45(1), 70-95. https://doi.org/10.1177/0149206318793196

Sastre-Castillo, M. A., Peris-Ortiz, M. \& Danvila-Del Valle, I. (2015). What is different about the profile of the social entrepreneur? Nonprofit Management \& Leadership, 25(4), 349-369. https://doi.org/10.1002/nml.21138

Savitz, A. W. \& Weber, K. (2006). The triple bottom line: How today's best-run companies are achieving economic, social and environmental success - and how you can too. Jossey-Bass.

Schwartz, S. H. (1992). Universals in the content and structure of values: Theoretical advances and empiric al tests in 20 countries. En M. P. Zanna (Ed.), Advances in experimental social psychology (Vol. 25, pp. 1-65). Academic Press. https://doi.org/10.1016/S0065$\underline{2601(08) 60281-6}$

Schwartz, S. H. (1994). Are there universal aspects in the structure and contents of human values? Journal of Social Issues, 50(4), 1945. https://doi.org/10.1111/j.1540-4560.1994.tb01196.x

Schwartz, S. H. (2010). Basic values: How they motivate and inhibit prosocial behavior. En M. Mikulincer \& P. R. Shaver (Eds.), Prosocial motives, emotions, and behavior: The better angels of our nature (pp. 221-241). American Psychological Association. https://doi.org/10.1037/12061-012

Schwartz, S. H. \& Bilsky, W. (1987). Toward a universal psychological structure of human values. Journal of Personality and Social Psychology, 53(3), 550-562. https://doi.org/10.1037/0022-3514.53.3.550

Seelos, C. \& Mair, J. (2005). Social entrepreneurship: Creating new business models to serve the poor. Business Horizons, 48(3), 241246. https://doi.org/10.1016/j.bushor.2004.11.006

Serida, J., Nakamatsu, K. \& Uehara, L. (2010). Global Entrepreneurship Monitor Perú 2008. Esan Ediciones.

Shaver, K. G. \& Scott, L. R. (1992). Person, process, choice: The psychology of new venture creation. Entrepreneurship Theory and Practice, 16(2), 23-46. https://doi.org/10.1177/104225879201600204

Smith, C., Smith, J. B. \& Shaw, E. (2017). Embracing digital networks: Entrepreneurs' social capital online. Journal of Business Venturing, 32(1), 18-34. https://doi.org/10.1016/j.jbusvent.2016.10.003

Sullivan Mort, G., Weerawardena, J. \& Carnegie, K. (2003). Social entrepreneurship: Towards conceptualisation. International Journal of Nonprofit and Voluntary Sector Marketing, 8(1), 76-88. https://doi.org/10.1002/nvsm.202

Vera Ruiz, A. (2019). Perfiles psicológicos de emprendedores en Perú según tipos de origen y valor. [Tesis de doctorado no publicada]. Pontificia Universidad Católica del Perú.

Vera Ruiz, A., Prialé Valle, M. A., Espinosa Pezzia, A. \& Ninahuanca López, E. F. (2019). Narrativas de sostenibilidad en emprendedores sociales de Lima Metropolitana. Summa Psicológica UST, 16(2), 88-97. https://doi.org/10.18774/0719-448.x2019.16.421

Vera Ruiz, A., Prialé Valle, M. A., Espinosa Pezzia, A. \& Ninahuanca López, E. F. (2020). Modelando una propuesta conceptual para analizar características psicosociales de emprendedores sociales peruanos. LIBERABIT: Revista Peruana de Psicología, 26(1), Artículo e256. https://doi.org/10.24265/liberabit.2020.v26n1.03

Whitman, J. (2011). Social entrepreneurship: An overview. En W. Bygrave \& A. Zacharakis (Eds.), Entrepreneurship: Second edition (pp. 563-595). John Wiley \& Sons. 
Wood, S. (2012). Prone to progress: Using personality to identify supporters of innovative social entrepreneurship. Journal of Public Policy \& Marketing, 31(1), 129-141. https://doi.org/10.1509/jppm.11.060

Young, R. (2006). For what it is worth: Social value and the future of social entrepreneurship. En A. Nicholls (Ed.), Social entrepreneurship: New models of sustainable social change (pp. 56-73). Oxford University Press.

Zahra, S. A., Gedajlovic, E., Neubaum, D. O. \& Shulman, J. M. (2009). A typology of social entrepreneurs: Motives, search processes and ethical challenges. Journal of Business Venturing, 24(5), 519-532. https://doi.org/10.1016/j.jbusvent.2008.04.007

Zhang, D. D. \& Swanson, L. A. (2014). Linking social entrepreneurship and sustainability. Journal of Social Entrepreneurship, 5(2), 175-191. https://doi.org/10.1080/19420676.2014.880503

Zhao, H. \& Seibert, S. E. (2006). The big five personality dimensions and entrepreneurial status: A meta-analytical review. Journal of Applied Psychology, 91(2), 259-271. https://doi.org/10.1037/0021-9010.91.2.259

Fecha de recepción: Abril de 2019.

Fecha de aceptación: Octubre de 2020. 